\title{
STABILITY OF MULTIPLIERS ON BANACH ALGEBRAS
}

\author{
TAKESHI MIURA, GO HIRASAWA, and SIN-EI TAKAHASI
}

Received 2 February 2004

\begin{abstract}
Suppose $A$ is a Banach algebra without order. We show that an approximate multiplier $T$ : $A \rightarrow A$ is an exact multiplier. We also consider an approximate multiplier $T$ on a Banach algebra which need not be without order. If, in addition, $T$ is approximately additive, then we prove the Hyers-Ulam-Rassias stability of $T$.
\end{abstract}

2000 Mathematics Subject Classification: 46J10.

1. Introduction and statement of results. It seems that the stability problem of functional equations had been first raised by Ulam (cf. [5, Chapter VI] and [6]): for what metric groups $G$ is it true that a $\varepsilon$-automorphism of $G$ is necessarily near to a strict automorphism?

An answer to the above problem has been given as follows. Suppose $E_{1}$ and $E_{2}$ are two real Banach spaces and $f: E_{1} \rightarrow E_{2}$ is a mapping. If there exist $\delta \geq 0$ and $p \geq 0$, $p \neq 1$, such that

$$
\|f(x+y)-f(x)-f(y)\| \leq \varepsilon\left(\|x\|^{p}+\|y\|^{p}\right)
$$

for all $x, y \in E_{1}$, then there is a unique additive mapping $T: E_{1} \rightarrow E_{2}$ such that $\| f(x)-$ $T(x)\|\leq 2 \varepsilon\| x \|^{p} /\left|2-2^{p}\right|$ for every $x \in E_{1}$. This result is called the Hyers-Ulam-Rassias stability of the additive Cauchy equation $g(x+y)=g(x)+g(y)$. Indeed, Hyers [2] obtained the result for $p=0$. Then Rassias [3] generalized the above result of Hyers to the case where $0 \leq p<1$. Gajda [1] solved the problem for $1<p$, which was raised by Rassias. In the same paper, Gajda also gave an example that a similar result does not hold for $p=1$. We can also find another example in [4]. If $p<0$, then $\|x\|^{p}$ is meaningless for $x=0$. In this case, if we assume that $\|0\|^{p}$ means $\infty$, then the proof given in [3] shows the existence of a mapping $T: E_{1} \backslash\{0\} \rightarrow E_{2}$ such that $\|f(x)-T(x)\| \leq$ $2 \varepsilon\|x\|^{p} /\left|2-2^{p}\right|$ for every $x \in E_{1} \backslash\{0\}$. Moreover, if we define $T(0)=0$, then we see that the extended mapping, denoted by the same letter $T$, is additive. The last inequality is valid for $x=0$ since we assume $\|0\|^{p}=\infty$. Thus, the Hyers-Ulam-Rassias stability holds for $p \in \mathbb{R} \backslash\{1\}$, where $\mathbb{R}$ denotes the real number field.

Suppose $A$ is a Banach algebra. We say that a mapping $T: A \rightarrow A$ is a multiplier if $a(T b)=(T a) b$ for all $a, b \in A$. Recall that a Banach algebra $A$ is not without order if there exist $x_{0}, y_{0} \in A \backslash\{0\}$ such that $x_{0} A=A y_{0}=\{0\}$. Therefore, $A$ is without order if and only if for all $x \in A, x A=\{0\}$ implies $x=0$, or, for all $x \in A, A x=\{0\}$ implies $x=0$. We first prove the superstability of multipliers on a Banach algebra without order; that is, each approximate multiplier is an exact multiplier. 
THeOREM 1.1. Suppose $A$ is a complex Banach algebra without order. If $T: A \rightarrow A$ is a mapping such that

$$
\|a(T b)-(T a) b\| \leq \varepsilon\|a\|^{p}\|b\|^{p} \quad(a, b \in A)
$$

for some $\varepsilon \geq 0$ and $p \geq 0, p \neq 1$, then $T$ is a multiplier.

In Theorem 1.1, we only consider the case where $p \geq 0, p \neq 1$. Even if $p<0$, we can also obtain a result similar to Theorem 1.1 under an additional but natural assumption that $T(0)=0$.

THEOREM 1.2. Suppose $A$ is a complex Banach algebra without order and suppose $T: A \rightarrow A$ is a mapping such that $T(0)=0$ and $\|a(T b)-(T a) b\| \leq \varepsilon\|a\|^{p}\|b\|^{p}(a, b \in A)$ for some $\varepsilon \geq 0$ and $p<0$, where $\|0\|^{p}$ means $\infty$. Then $T$ is a multiplier.

Theorem 1.1 need not be true for $p=1$. In fact, in Remark 2.1, we give an approximate multiplier which is not an exact multiplier; however, in Remark 2.2, we see that the Hyers-Ulam-Rassias stability holds for approximate multipliers between unital commutative Banach algebras.

If $A$ is a Banach algebra which need not be without order, then under an additional assumption, we show the Hyers-Ulam-Rassias stability of multiplier on $A$ : if $f$ is an approximate multiplier which is also approximately additive, then there is a multiplier near to $f$.

THEOREM 1.3. Suppose $A$ is a Banach algebra, which need not be without order, and $f: A \rightarrow A$ is a mapping such that

$$
\begin{gathered}
\|f(a+b)-f(a)-f(b)\| \leq \varepsilon\left(\|a\|^{p}+\|b\|^{p}\right) \quad(a, b \in A), \\
\|a f(b)-f(a) b\| \leq \varepsilon\|a\|^{p}\|b\|^{p} \quad(a, b \in A)
\end{gathered}
$$

for some $\varepsilon \geq 0$ and $p \in \mathbb{R}$. If $p \geq 0$ and $p \neq 1$, or $p<0$ and $f(0)=0$, then there is $a$ multiplier $T: A \rightarrow A$ such that

$$
\|f(a)-T a\| \leq \frac{2 \varepsilon}{\left|2-2^{p}\right|}\|a\|^{p} \quad(a \in A) .
$$

\section{Proofs of the results}

Proof of Theorem 1.1. We first show that $T$ is homogeneous, that is, $T(\lambda a)=$ $\lambda T a$ for all $\lambda \in \mathbb{C}$ and $a \in A$. To do this, pick $\lambda \in \mathbb{C}, a \in A$ and fix $x \in A$ arbitrarily. Put $s=(1-p) /|1-p|$. For each $n \in \mathbb{N}$, it follows from (1.2) that

$$
\begin{aligned}
\left\|n^{s} x[T(\lambda a)-\lambda T a]\right\| \leq & \left\|n^{s} x[T(\lambda a)]-\left[T\left(n^{s} x\right)\right](\lambda a)\right\| \\
& +\left\|\left[T\left(n^{s} x\right)\right](\lambda a)-n^{s} x(\lambda T a)\right\| \\
\leq & \varepsilon\left\|n^{s} x\right\|^{p}\|\lambda a\|^{p}+|\lambda| \varepsilon\left\|n^{s} x\right\|^{p}\|a\|^{p} \\
\leq & n^{s p} \varepsilon\left(|\lambda|^{p}+|\lambda|\right)\|x\|^{p}\|a\|^{p}
\end{aligned}
$$


and hence

$$
\|x[T(\lambda a)-\lambda T a]\| \leq n^{s(p-1)} \varepsilon\left(|\lambda|^{p}+|\lambda|\right)\|x\|^{p}\|a\|^{p}
$$

for all $n \in \mathbb{N}$. Since $s(p-1)<0$, we obtain by letting $n \rightarrow \infty$ in (2.2) that $x[T(\lambda a)-$ $\lambda T a]=0$. Similarly to the argument above, we can also get $[T(\lambda a)-\lambda T a] x=0$. Since $A$ is without order, we conclude that $T(\lambda a)=\lambda T a$, which implies the homogeneity of $T$.

Now we are ready to prove that $T$ is a multiplier. Since $T$ is homogeneous, $T(a)=$ $n^{-s} T\left(n^{s} a\right)$ for all $n \in \mathbb{N}$. Recall that, by definition, $s(p-1)<0$. We thus obtain for all $a, b \in A$,

$$
\begin{aligned}
\|a(T b)-(T a) b\| & =n^{-s}\left\|n^{s} a(T b)-T\left(n^{s} a\right) b\right\| \\
& \leq n^{-s} \varepsilon\left\|n^{s} a\right\|^{p}\|b\|^{p}=n^{s(p-1)} \varepsilon\|a\|^{p}\|b\|^{p} \\
& \longrightarrow 0 \quad \text { as } n \longrightarrow \infty .
\end{aligned}
$$

Hence $a(T b)=(T a) b$, proving $T$ is a multiplier.

Proof of Theorem 1.2. Since $T(0)=0$, it suffices to show that $a(T b)=(T a) b$ for all $a, b \in A \backslash\{0\}$. So, fix $a, b \in A \backslash\{0\}$ arbitrarily. In this case, inequalities (2.1) and (2.2) are also valid for $p<0$. Recall that we assume $\|0\|^{p}=\infty$, and hence

$$
x[T(\lambda a)-\lambda T a]=0, \quad[T(\lambda a)-\lambda T a] x=0,
$$

for $\lambda \in \mathbb{C} \backslash\{0\}$ and $x \in A \backslash\{0\}$. Note that (2.4) is also true for $x=0$. Since $A$ is without order, we thus obtain $T(\lambda a)=\lambda T a$ for all $\lambda \in \mathbb{C} \backslash\{0\}$. An argument similar to (2.3) shows $a(T b)=(T a) b$, and the proof is complete.

REMARK 2.1. A result similar to Theorem 1.1 need not be true for $p=1$, that is, there exists an approximate multiplier which is not an exact multiplier. More explicitly, to each $\varepsilon>0$ there corresponds a function $f: \mathbb{C} \rightarrow \mathbb{C}$ which is not a multiplier such that

$$
\left|z_{1} f\left(z_{2}\right)-f\left(z_{1}\right) z_{2}\right| \leq \varepsilon\left|z_{1}\right|\left|z_{2}\right|
$$

for all $z_{1}, z_{2} \in \mathbb{C}$. Fix $\varepsilon>0$ arbitrarily. By the continuity of the function $t \mapsto e^{i t}$, there corresponds a $\delta$ with $0<\delta<1$ such that $|t|<2 \pi(1-\delta)$ implies $\left|e^{i t}-1\right|<\varepsilon$. With this $\delta$, we define the mapping $f: \mathbb{C} \rightarrow \mathbb{C}$ by

$$
f(z)= \begin{cases}0 & z=0, \\ |z| e^{i \delta \theta} & z \in \mathbb{C} \backslash\{0\},\end{cases}
$$

where $\theta \in[0,2 \pi)$ denotes the argument of $z$. Then we see that $f$ satisfies inequality (2.5) for all $z_{1}, z_{2} \in \mathbb{C}$. Since the case where $z_{1}=0$ or $z_{2}=0$ is trivial, we only consider $z_{1}, z_{2} \in \mathbb{C} \backslash\{0\}$. If $z_{j}=\left|z_{j}\right| e^{i \theta_{j}}$ for $j=1,2$, then we get

$$
\left|z_{1} f\left(z_{2}\right)-f\left(z_{1}\right) z_{2}\right|=\left|z_{1}\right|\left|z_{2}\right|\left|e^{i(1-\delta)\left(\theta_{1}-\theta_{2}\right)}-1\right| .
$$

Note that $\left|\theta_{1}-\theta_{2}\right|<2 \pi$. By the definition of $\delta$, we obtain (2.5), which implies that $f$ is an approximate multiplier. Moreover, $f$ is not an exact multiplier, and hence Theorem 1.1 does not hold for $p=1$ in general. 
REMARK 2.2. Suppose $A$ is a unital commutative Banach algebra. If $f: A \rightarrow A$ is a mapping such that

$$
\|a f(b)-f(a) b\| \leq \varepsilon\|a\|\|b\| \quad(a, b \in A)
$$

for some $\varepsilon \geq 0$, then there is an exact multiplier $T: A \rightarrow A$ such that

$$
\|f(a)-T a\| \leq \varepsilon\|a\| \quad(a \in A) .
$$

Indeed, let $e \in A$ be a unit element. Taking $b=e$ in (2.8), we obtain

$$
\|a f(e)-f(a)\| \leq \varepsilon\|a\| \quad(a \in A) .
$$

If we consider the mapping $T: A \rightarrow A$ defined by

$$
T a=a f(e) \quad(a \in A)
$$

then $T$ is a multiplier such that $\|f(a)-T a\| \leq \varepsilon\|a\|$ for all $a \in A$.

Proof of Theorem 1.3. Suppose $p \neq 1$. By (1.3), it follows from a theorem of Rassias [3] and Gajda [1] that there exists a unique additive mapping $T: A \rightarrow A$ such that (1.5) holds. So, we need to show that $a(T b)=(T a) b$ for all $a, b \in A$. Since $T$ is additive, $T(0)=0$, and hence it is enough to consider $a, b \in A \backslash\{0\}$. Put $s=(1-p) /|1-p|$ and fix $a, b \in A \backslash\{0\}$ arbitrarily. Since $T$ is additive, we see that $T a=n^{-s} T\left(n^{s} a\right)$ for each $n \in \mathbb{N}$. Now it follows from (1.5) that

$$
\left\|n^{-s} f\left(n^{s} b\right)-T b\right\| \leq n^{-s} \frac{2 \varepsilon}{\left|2-2^{p}\right|}\left\|n^{s} b\right\|^{p}=n^{s(p-1)} \frac{2 \varepsilon}{\left|2-2^{p}\right|}\|b\|^{p}
$$

for all $n \in \mathbb{N}$, and hence

$$
\left\|n^{-s} f\left(n^{s} b\right)-T b\right\| \rightarrow 0 \text { as } n \rightarrow \infty .
$$

Since $f$ is an approximate multiplier, we get

$$
\begin{aligned}
\left\|n^{-s} a f\left(n^{s} b\right)-f(a) b\right\| & =n^{-s}\left\|a f\left(n^{s} b\right)-f(a) n^{s} b\right\| \\
& \leq n^{-s} \varepsilon\|a\|^{p}\left\|n^{s} b\right\|^{p} \\
& =n^{s(p-1)} \varepsilon\|a\|^{p}\|b\|^{p}
\end{aligned}
$$

for all $n \in \mathbb{N}$. Hence,

$$
\left\|n^{-s} a f\left(n^{s} b\right)-f(a) b\right\| \longrightarrow 0 \quad \text { as } n \longrightarrow \infty .
$$

Now it follows from (2.13) and (2.15) that

$$
\begin{aligned}
& \|a(T b)-(T a) b\| \\
& \quad \leq\|a\|\left\|T b-n^{-s} f\left(n^{s} b\right)\right\|+\left\|n^{-s} a f\left(n^{s} b\right)-f(a) b\right\|+\|f(a) b-(T a) b\| \\
& \quad \longrightarrow\|f(a) b-(T a) b\| \text { as } n \longrightarrow \infty .
\end{aligned}
$$


By (1.5), we obtain

$$
\|a(T b)-(T a) b\| \leq \frac{2 \varepsilon}{\left|2-2^{p}\right|}\|a\|^{p}\|b\| .
$$

An argument similar to (2.3) implies $\|a(T b)-(T a) b\|=0$, proving $T$ is a multiplier.

\section{REFERENCES}

[1] Z. Gajda, On stability of additive mappings, Int. J. Math. Math. Sci. 14 (1991), no. 3, 431-434.

[2] D. H. Hyers, On the stability of the linear functional equation, Proc. Nat. Acad. Sci. U. S. A. 27 (1941), 222-224.

[3] Th. M. Rassias, On the stability of the linear mapping in Banach spaces, Proc. Amer. Math. Soc. 72 (1978), no. 2, 297-300.

[4] Th. M. Rassias and P. Šemrl, On the behavior of mappings which do not satisfy Hyers-Ulam stability, Proc. Amer. Math. Soc. 114 (1992), no. 4, 989-993.

[5] S. M. Ulam, A Collection of Mathematical Problems, Interscience Tracts in Pure and Applied Mathematics, no. 8, Interscience Publishers, New York, 1960.

[6] _ Sets, Numbers, and Universes: Selected Works, Mathematicians of Our Time, vol. 9, MIT Press, Massachusetts, 1974, edited by W. A. Beyer, J. Mycielski, and G.-C. Rota.

Takeshi Miura: Department of Basic Technology, Yamagata University, Yonezawa 992-8510, Japan

E-mail address: mi ura@yz.yamagata-u.ac.jp

Go Hirasawa: Department of Mathematics, Nippon Institute of Technology, Miyashiro, Saitama 345-8501, Japan

E-mail address: hi rasawa1@muh.big1obe.ne.jp

Sin-Ei Takahasi: Department of Basic Technology, Yamagata University, Yonezawa 992-8510, Japan

E-mail address: sin-ei@emperor.yz.yamagata-u.ac.jp 


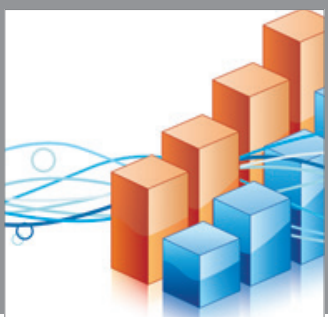

Advances in

Operations Research

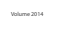

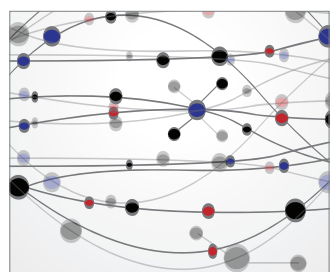

\section{The Scientific} World Journal
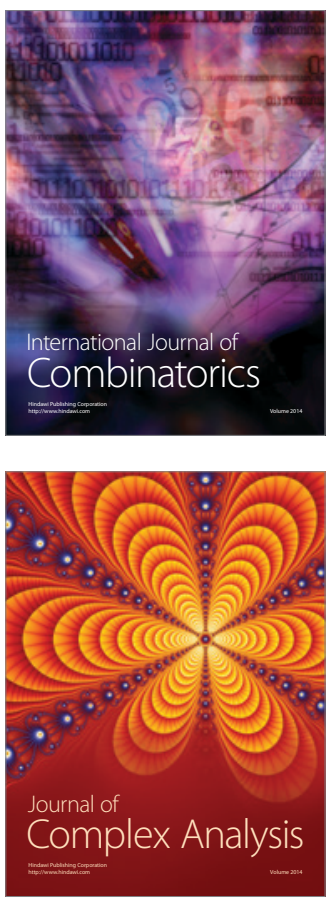

International Journal of

Mathematics and

Mathematical

Sciences
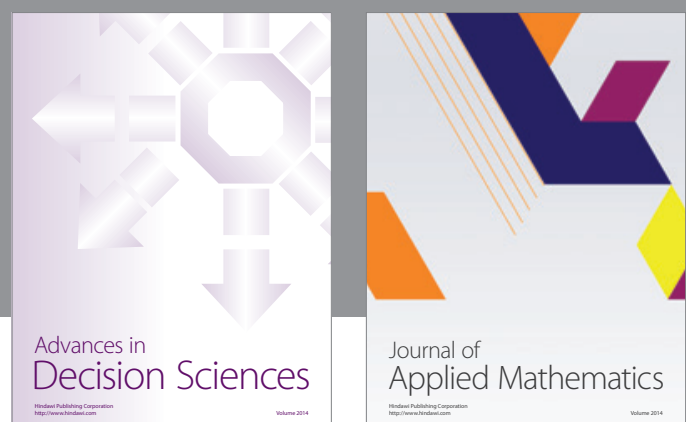

Journal of

Applied Mathematics
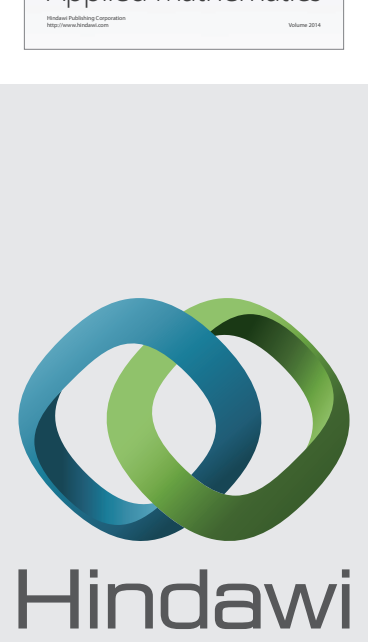

Submit your manuscripts at http://www.hindawi.com
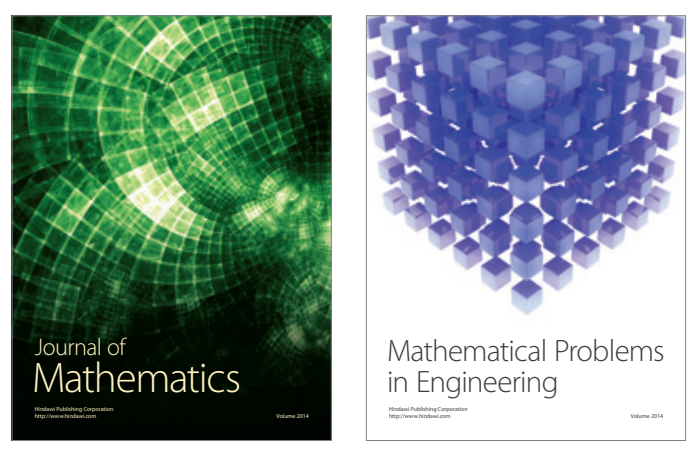

Mathematical Problems in Engineering
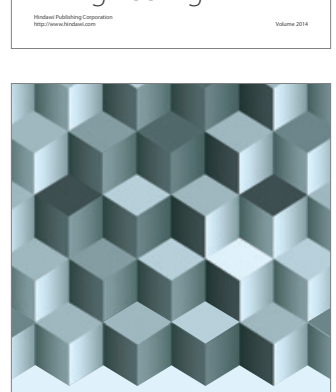

Journal of

Function Spaces
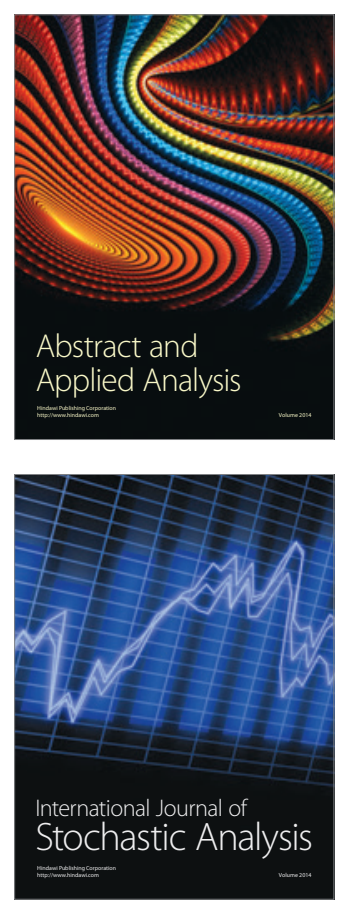

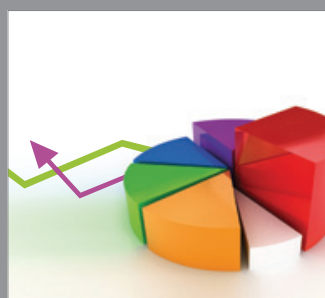

ournal of

Probability and Statistics

Promensencen
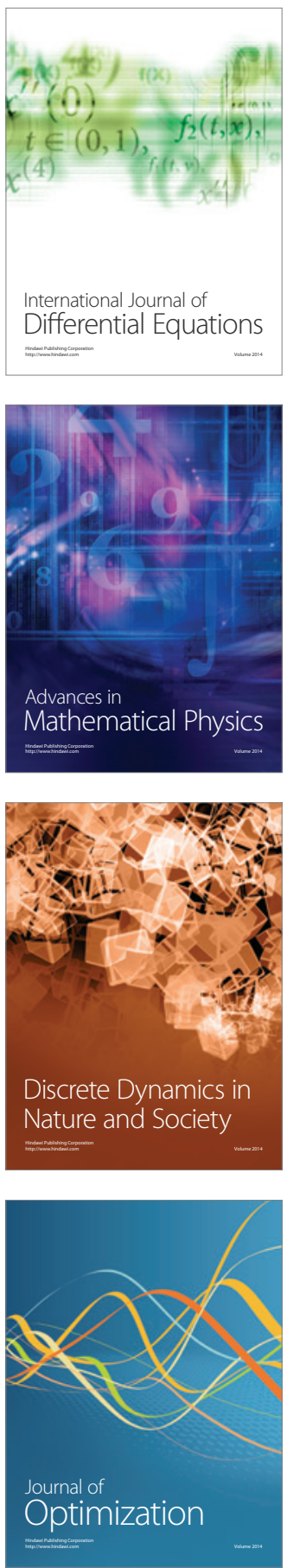